\title{
Generalized equivalent circuits for mixed conductors: silver sulfide as a model system
}

\author{
Jong-Sook Lee $\cdot$ Janez Jamnik $\cdot$ Joachim Maier
}

Received: 10 November 2008/ Accepted: 22 December 2008/Published online: 19 March 2009

(C) Springer-Verlag 2009

\begin{abstract}
The generalized equivalent circuit for HebbWagner polarization in the frequency domain proposed by Jamnik and Maier (J Electrochem Soc 146:4183, 1999) includes the space-charge polarization that was previously neglected. In the present work, using a self-coded Fortran program, the completely generalized equivalent circuit is successfully applied to a mixed conducting silver sulfide with an AgI electrode that suppresses the electronic flow. A whole set of fit parameters, such as geometric capacitance, partial conductivities, chemical capacitance or diffusivity, and the blocking and shunting characteristics of electrodes are independently but self-consistently obtained over a range of silver activities, as controlled by a galvanic cell. The interfacial capacitance was found to be much larger than the diffuse space-charge double-layer capacitance and was thus ascribed to the adsorption capacitance at the core of the interface, which should be connected in parallel with the space-charge double-layer polarization. Two simplified equivalent circuits were shown to be good approximations for the spectra at the extreme low and high silver activity, respectively.
\end{abstract}

It was belatedly learned that Derek Johnson, Scribner Ass. Inc., has implemented a "Jamink+Maier/Mixed Conductor" model in Zview 3.1.

J.-S. Lee $(\square)$

School of Materials Science and Engineering,

Chonnam National University, Gwangju 500-757, Korea

e-mail: jongsook@jnu.ac.kr

J. Jamnik

National Institute of Chemistry, 1000 Ljubljana, Slovenia

J. Maier

Max-Planck-Institut für Festkörperforschung,

70569 Stuttgart, Germany
Keywords Impedance spectroscopy - Mixed conductor . Chemical diffusion - Hebb-Wagner polarization . Space-charge polarization - Adsorption capacitance

\section{Introduction}

All ionic crystals, whether they are predominantly electronically conducting or mostly ionically conducting, can be considered mixed conductors of different degrees. It goes without saying that this mixed ionic-electronic conduction plays critical functional roles in solid state electrochemical devices such as fuel cells, batteries, electrochemical sensors and electrochromic devices. Mixed ionic-electronic conduction is also one of the hot issues in state-of-the-art electronics. Ionic conduction in dielectric materials and oxide semiconductors is responsible for the degradation of electronic components and devices such as multilayer capacitors, varistors and oxide transistors. Therefore, the proper characterization of mixed conduction phenomena is of the utmost importance to a broad field of applications.

Mixed ionic-electronic conduction in $\mathrm{Ag}_{2} \mathrm{~S}$ has drawn considerable attention as a working principle for the recently demonstrated Ag nanoswitch [1]. Silver sulfide was in fact the first "semiconducting" material named by Faraday more than 170 years ago; it was described as an "extraordinary case" that exhibited conductivity with a positive temperature coefficient [2]. Significant ionic conduction was later found to be present $[3,4] . \mathrm{Ag}_{2} \mathrm{~S}$ exhibits representative mixed conduction behavior: the electronic transference number $\left(t_{\text {eon }}\right)$ varies from $\sim 0.5$ to 1 with increasing $\mathrm{Ag}$ activity $\left(a_{\mathrm{Ag}}\right)$ at $160{ }^{\circ} \mathrm{C}$, and $a_{\mathrm{Ag}}$ can be easily controlled by a coulometric titration cell such as $\mathrm{Pt}\left|\mathrm{Ag}_{2} \mathrm{~S}\right| \mathrm{AgI} \mid \mathrm{Ag}$; for high-temperature modification of 


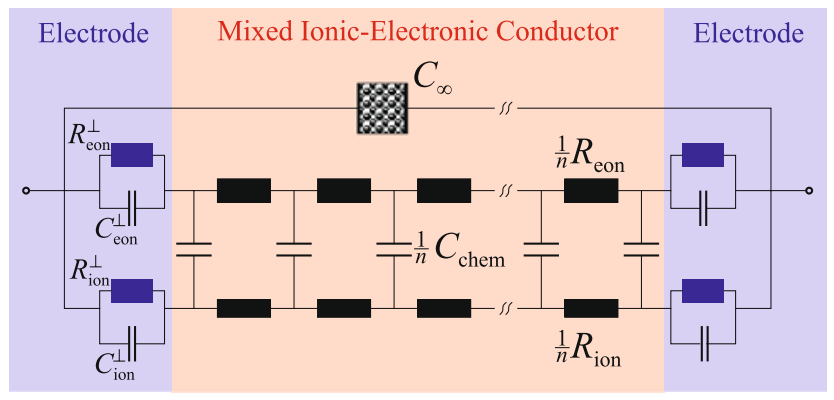

Fig. 1 Generalized equivalent circuit for a mixed conductor with a thickness that is much greater than the Debye length $\lambda$. The quantities $R_{\text {ion }}, R_{\text {eon }}$, and $C_{\text {chem }}$, which all refer to the overall sample, are weighted by $1 / n$, where $n$ is the number of transmission line unit cells [16]

$\mathrm{Ag}_{2} \mathrm{~S}\left(>176^{\circ} \mathrm{C}\right)$ the conduction electrons are degenerate and $t_{\text {eon }} \simeq 1$ in the whole stability range [5]. It is surprising, however, that a comprehensive study of the transport properties of such an ideal model system is not yet available. Partial conductivities [5, 6] and chemical diffusion coefficients $[7,8]$ were reported separately.

The mixed conduction in silver sulfide semiconductors has been studied by using electrodes that selectively block electrons or ions [4, 5, 9-13]. Stoichiometry polarization is induced across the specimen by the blocked charge species, and the transport of the unblocked species in the material is thus transported by chemical diffusion under the activity gradient (rather than conduction being driven by the electric field). Therefore, the time dependence of polarization or depolarization allows the chemical diffusion coefficient $\left(D_{\text {chem }}\right)$ as well as the partial ionic and electronic conductivities $\left(\sigma_{\text {ion }}, \sigma_{\text {eon }}\right)[12]$ to be determined.

The polarization behavior in the time domain can be equivalently represented in the frequency domain by a Fourier-Laplace transformation, which can be elegantly and conveniently modeled by circuit elements such as resistors and capacitors [14, 15]. Even the chemical diffusion resulting from the stoichiometry polarization can be represented by a transmission line model or Warburg impedance, where the ionic and electronic conduction paths are shunted by the chemical capacitance $C_{\text {chem }}$, representing the ability of the sample $\left(\mathrm{Ag}_{2} \mathrm{~S}\right)$ to store chemical energy [16]. The chemical capacitance is inversely proportional to the thermodynamic factor. Previously, such full spectra have been constructed by combining impedance spectra measured directly using a LCR meter in the high frequency range $(>5 \mathrm{~Hz})$ with the low frequency data obtained by a Fourier-Laplace transformation of dc relaxation experiments [15, 17, 18].

The generalized circuit $[16,19,20]$ shown in Fig. 1 explicitly includes the blocking effects of the electrodes, in addition to the material parameters of the mixed conductor described above. The material and physical properties represented by the circuit elements are indicated in Table 1. It should be noted that the resistance and capacitance of the electrodes $R_{\text {ion(eon) }}^{\perp}$, and $C_{\text {ion(eon) }}^{\perp}$ refer to the functionality with respect to the mixed conductor, not to the property of the electrode material itself. For ideally reversible and blocking electrodes, $R_{\text {ion(eon) }}^{\perp}$ is 0 and $\infty$, respectively. The capacitance $C_{\text {ion(eon) }}^{\perp}$ is the electrostatic polarization defined by the Debye screening length, $\lambda_{\text {ion(eon) }} \equiv \sqrt{\epsilon \epsilon_{0} k_{\mathrm{B}} T /\left(z_{\text {ion(eon })}^{2} e^{2} c_{\text {ion(eon })}\right)}$. This may be considered a "key signature" that distinguishes electrochemical Hebb-Wagner polarization from purely chemically induced relaxation. This model has been explicitly or implicitly applied [21-25].

In the present work, for the first time, the generalized equivalent circuit in its complete form is applied to a Hebb-Wagner polarization cell with silver sulfide used as a model mixed conductor. All of the independent fit
Table 1 Relationship between the circuit elements in Fig. 1 and the material parameters and fit results for the spectra in Fig. 2

The units are $\Omega, F(\operatorname{arad})$, and $\mathrm{H}$ (enry), respectively, for resistance, capacitance, and inductance

\begin{tabular}{|c|c|c|c|c|}
\hline \multicolumn{2}{|l|}{ Parameters } & \multicolumn{3}{|l|}{ Fit results } \\
\hline \multirow[t]{2}{*}{ Model parameters } & \multirow{2}{*}{$\begin{array}{l}\text { Material and physical } \\
\text { parameters }\end{array}$} & \multicolumn{3}{|c|}{$\mathrm{EMF}$ of $\mathrm{Pt} / \mathrm{Ag} / \mathrm{AgI} / \mathrm{Ag}_{2} \mathrm{~S} / \mathrm{Pt}$} \\
\hline & & $10 \mathrm{mV}$ & $80 \mathrm{mV}$ & $140 \mathrm{mV}$ \\
\hline$R_{\text {ion }}$ & $\sigma_{\text {ion } \frac{L}{A}}^{-1}$ & $0.73755 \times 10^{4}$ & $0.71358 \times 10^{4}$ & $0.67112 \times 10^{4}$ \\
\hline$R_{\text {eon }}$ & $\sigma_{\text {eon }}^{-1} \frac{L}{A}$ & $0.13908 \times 10^{3}$ & $0.80051 \times 10^{3}$ & $0.45081 \times 10^{4}$ \\
\hline$R_{\text {ion }}^{\perp}$ & 0 or $\infty$ & $0.20314 \times 10^{-7}$ & $0.15307 \times 10^{-7}$ & $0.23938 \times 10^{-7}$ \\
\hline$R_{\text {eon }}^{\perp}$ & 0 or $\infty$ & $0.97407 \times 10^{21}$ & $0.74765 \times 10^{22}$ & $0.85435 \times 10^{23}$ \\
\hline$C_{\infty}$ & $\epsilon \epsilon_{0} \frac{A}{L}$ & $0.73707 \times 10^{-10}$ & $0.18049 \times 10^{-10}$ & $0.12489 \times 10^{-10}$ \\
\hline$C_{\text {chem }}$ & $\frac{L^{2}}{\left(R_{\text {ogp }}+R_{\text {eоp }}\right) D_{\text {chem }}}$ & $0.10529 \times 10^{-1}$ & $0.47033 \times 10^{-2}$ & $0.58395 \times 10^{-2}$ \\
\hline$C_{\mathrm{ion}}^{\perp}$ & $\epsilon \epsilon_{0} \frac{A}{\lambda_{\text {inp }}}$ & $0.19840 \times 10^{-3}$ & $0.11244 \times 10^{-2}$ & $0.15971 \times 10^{-1}$ \\
\hline$C_{\mathrm{eon}}^{\perp}$ & $\epsilon \epsilon_{0} \frac{A}{\lambda_{e 00}}$ & $0.71793 \times 10^{-4}$ & $0.46967 \times 10^{-5}$ & $0.19817 \times 10^{-6}$ \\
\hline Inductance & Lead wire & $0.34853 \times 10^{-5}$ & $0.56928 \times 10^{-5}$ & $0.77201 \times 10^{-5}$ \\
\hline Sum of squares & & 0.16760 & $0.38197 \times 10^{-1}$ & $0.22538 \times 10^{-1}$ \\
\hline
\end{tabular}


parameters obtained using the equivalent circuit lead to self-consistent physicochemical parameters of the experimental electrochemical cell.

\section{Experimental}

A symmetric cell, $\mathrm{Ag}|\mathrm{AgI}| \mathrm{Ag}_{2} \mathrm{~S}|\mathrm{AgI}| \mathrm{Ag}$, was constructed and impedance was measured at $160{ }^{\circ} \mathrm{C}$ in the frequency range from $10^{7}$ to $\sim 10^{-3} \mathrm{~Hz}$ using a Solartron 1260 (UK, Schlumberger). The measurement was performed from high to low frequency. Powder compacts of $\mathrm{Ag}_{2} \mathrm{~S}$ (Aldrich, 99.9\%) and AgI (Aldrich, 99.999\%) were cold isostatically pressed and heat treated at $300{ }^{\circ} \mathrm{C}$ for one day. A galvanic cell, Pt|Ag 2 SlAgI|Ag, using Pt wire wound around a $\mathrm{Ag}_{2} \mathrm{~S}$ specimen [area $(A) 0.2 \times 0.19 \mathrm{~cm}^{2}$; length $(L) 1.5 \mathrm{~cm}$ ] controlled the silver activity $\left(a_{\mathrm{Ag}}\right)$ by applying the potential $\mathrm{EMF}=-(\mathrm{RT} / \mathrm{F}) \ln a_{\mathrm{Ag}}$ with respect to Ag using a constant voltage source (Keithley 228) before each impedance measurement until the material was fully equilibrated within the stability range with EMF $<150 \mathrm{mV}$.

Fitting was performed using the complete equivalent circuit shown in Fig. 1 with the inductance element due to the lead wire added in series. If we skip the contribution of $C_{\infty}$ for the purpose of clarity, define interfacial impedances, $\quad Z_{\mathrm{ion}(\text { eon })}^{\perp} \equiv R_{\mathrm{ion}(\mathrm{eon})}^{\perp} /\left(1+i \omega R_{\mathrm{ion}(\text { eon })}^{\perp} C_{\mathrm{ion}(\text { eon })}^{\perp}\right), \quad$ and express the product $\left(R_{\text {ion }}+R_{\text {eon }}\right) C_{\text {chem }}$ by $D_{\text {chem }}$, the impedance can be expressed as:

$Z(\omega)=Z_{\infty}+\left(Z_{0}-Z_{\infty}\right) \frac{\left(1+\frac{R_{\text {ion }}+R_{\text {eon }}}{2\left(Z_{\text {ion }}^{\text {ion }}+Z_{\text {eon }}\right)}\right) \tanh \sqrt{\frac{i \omega L^{2}}{4 D_{\text {chem }}}}}{\sqrt{\frac{i \omega L^{2}}{4 D_{\text {chem }}}}+\frac{R_{\text {ion }}+R_{\text {eon }}}{2\left(Z_{\text {ion }}^{\perp}+Z_{\text {eon }}^{\perp}\right)} \tanh \sqrt{\frac{i \omega L^{2}}{4 D_{\text {chem }}}} .}$

The high and low frequency impedances, $Z_{\infty}$ and $Z_{0}$, are defined as

$$
\begin{aligned}
& Z_{\infty} \equiv \frac{R_{\text {ion }} R_{\text {eon }}}{R_{\text {ion }}+R_{\text {eon }}}+2 \frac{Z_{\text {ion }}^{\perp} Z_{\text {eon }}^{\perp}}{Z_{\text {ion }}^{\perp}+Z_{\text {eon }}^{\perp}}, \\
& \frac{1}{Z_{0}} \equiv \frac{1}{R_{\text {ion }}+2 Z_{\text {ion }}^{\perp}}+\frac{1}{R_{\text {eon }}+2 Z_{\text {eon }}^{\perp}} .
\end{aligned}
$$

The elegance and the beauty of the circuit lies in the interfacial capacitances, $C_{\mathrm{ion}}^{\perp}$ and $C_{\mathrm{eon}}^{\perp}$, defined individually as

$$
C_{\mathrm{ion}(\mathrm{eon})}^{\perp} \equiv \epsilon \epsilon_{0} \frac{A}{\lambda_{\mathrm{ion}(\mathrm{eon})}} ; \quad \lambda_{\text {ion (eon) }} \equiv \sqrt{\frac{\epsilon \epsilon_{0} k_{\mathrm{B}} T}{z_{\mathrm{ion}(\mathrm{eon})}^{2} e^{2} c_{\mathrm{ion}(\mathrm{eon})}}},
$$

which is precise for cases of $c_{\text {ion }} \gg c_{\text {eon }}$ or $c_{\text {ion }} \ll c_{\text {eon }}$, applicable for most situations in the solid state [16].

The analytical expression of Eq. 1 is obtained by arranging the transmission line elements into a tangent hyperbolic function, $R \tanh \sqrt{i \omega T} / \sqrt{i \omega T}$, a finite-length Warburg impedance with a short circuit terminus. Although the model itself is provided by currently available commercial and noncommercial fitting programs, a self-coded Fortran program is needed to fit all of the independent parameters that are intermixed among the coefficients and variables of the tangent hyperbolic functions as complex variables. Once the analytical expression is obtained, however, the fitting can be performed straightforwardly, by using the software programs capable of handling complex variables. A better fit was obtained when the sum of squares was minimized with each point normalized by its impedance magnitude rather than by real and imaginary impedance values, respectively, $[16,19,26]$, i.e.,

$S \equiv \sum_{\omega_{i=1}}^{\omega_{i=N}}\left[\frac{\operatorname{Re}\left(Z_{\exp }-Z_{\text {model }}\right)}{\left|Z_{\exp }\right|}\right]^{2}+\left[\frac{\operatorname{Im}\left(Z_{\exp }-Z_{\text {model }}\right)}{\left|Z_{\exp }\right|}\right]^{2}$.

The program is currently undergoing further development to provide the errors of the individual fit parameters as well.

\section{Results and discussion}

Figure 2 represents the experimental impedance spectra and the simulated spectra obtained using the fit results of Table 1 for $\mathrm{EMF}=10,80$, and $140 \mathrm{mV}$, respectively. The results show that the ionic conductivity corresponding to the low frequency limit is almost independent of $a_{\mathrm{Ag}}$, indicating high ionic disorder in the system. High frequency details indicate decreasing electronic conductivity with decreasing $a_{\mathrm{Ag}}$ (or increasing $a_{\mathrm{S}}$ ). The ionic transference numbers are estimated as 0.02, 0.10 and 0.40, respectively. The upper graphs in Fig. 3 represent the partial ionic and electronic conductivities and the transference numbers as a function of EMF. The ionic conductivity $\left(\sigma_{\text {ion }}\right)$ is essentially independent of the silver activity, indicating that intrinsic ionic defects are the dominant type of disorder in $\mathrm{Ag}_{2} \mathrm{~S}$. The electronic conductivity increases linearly with increasing $\mathrm{Ag}$ activity according to classical Boltzmann statistics. The absolute values and the activity dependence are consistent with the literature $[5,6]$.

The chemical diffusion coefficients estimated from $R_{\text {ion }}, R_{\text {eon }}$, and $C_{\text {chem }}$ (see Table 1) are 2.8, 6.0, $3.4 \times 10^{-2} \mathrm{~cm}^{2} \mathrm{~s}^{-1}$, respectively, for the spectra in Fig. 2 . The middle graphs in Fig. 3 represent the chemical capacitance or inverse thermodynamic factor and the chemical diffusivity as a function of Ag activity. According to the analysis described in [7], the maximum in the thermodynamic factor or the minimum in the chemical capacitance $C_{\text {chem }}$ corresponds to the stoichiometric point 

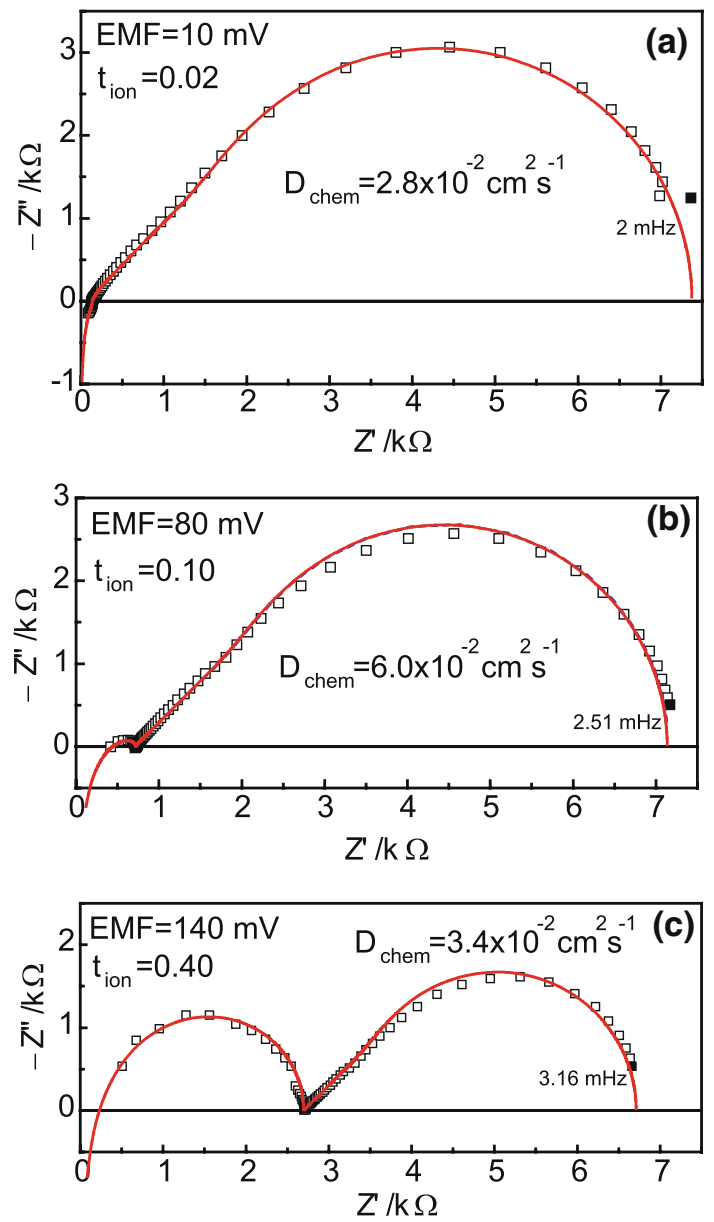

Fig. 2a-c Impedance spectra of $\mathrm{Ag}|\mathrm{AgI}| \mathrm{Ag}_{2} \mathrm{~S}|\mathrm{AgI}| \mathrm{Ag}$ when the EMF of the galvanic cell is $10 \mathrm{mV}$ (a), $80 \mathrm{mV}$ (b), and $140 \mathrm{mV}$ (c). The lines indicate the fit results obtained using the generalized equivalent circuit in Fig. 1

of $\operatorname{Ag}_{2+\delta} \mathrm{S}, \delta \approx 0$. Similar behavior has been reported for $\mathrm{Ag}_{2+\delta} \mathrm{Te}$ [25]. The decrease in electronic conductivity with sulfur activity over the whole measurement range can be explained by the higher mobility of electrons compared to holes. This shifts the chemical diffusivity maximum to a lower EMF value of ca. $82 \mathrm{mV}$.

Although the observed tendency is consistent with previous reports $[7,8]$, the chemical diffusivity maximum observed in our work appears much less pronounced than those in the previous works [there is also some difference in the temperatures $\left(160\right.$ vs. $\left.\left.168{ }^{\circ} \mathrm{C}[7,8]\right)\right]$. The line shown in the plot of the chemical capacitance is a fit to the theoretical cosine hyperbolic function [7, 27], however, shifted by a large constant of 0.0035 Farads. The stoichiometric point indicated by the minimum of the chemical capacitance is ca. $96 \mathrm{mV}$, which is substantially lower than the reported stoichiometric point [6] of ca. $120 \mathrm{mV}$. The reason for this discrepancy should be further investigated.
The lower graphs in Fig. 3 represent the functionality of selectively blocking electrodes. It should be emphasized that $\mathrm{Ag} \mid \mathrm{AgI}$ behaves as an almost ideal selectively blocking electrode under the present experimental conditions. The "fitted" $R_{\text {eon }}^{\perp}$ and $R_{\text {ion }}^{\perp}$ values are very large approaching $\infty$ and very small close to 0 , respectively. The bulk resistance of $\mathrm{AgI}$ in superionically conducting high-temperature modification, and the charge transfer impedance at the interfaces such as $\mathrm{Ag} \mid \mathrm{AgI}$ and $\mathrm{AgI} \mid \mathrm{Ag}_{2} \mathrm{~S}$, are all negligible as long as all of the cell components are sufficiently pressed together. This situation contrasts with analogous cells in the oxide system $[15,18,21,22]$, where the electrodes are hardly ideally selective because of either the gas phase reaction or the mixed conductivity of the electrode material itself. Moreover, there are often significantly large charge transfer reaction impedances that should be considered in addition to the elements considered in Fig. 1.

Probably the most decisive and essential element in the generalized equivalent circuit in Fig. 1 is the interfacial capacitance $C_{\text {ion(eon) }}^{\perp}$, which was omitted in earlier treatments $[14,15,18]$. It has been found that $C_{\text {eon }}^{\perp}$ for the blocking electrode increases as $\sqrt{a_{\mathrm{Ag}}}$ (lower left in Fig. 3), at least for the range that supposedly has a high reliability of fitting, as discussed later. The $C_{\text {ion }}^{\perp}$ values, though scattered, are more or less constant-ca. $0.007 \mathrm{~F}$ (not shown)—which is much larger than $C_{\text {eon }}^{\perp}$. This tendency is consistent with the defect structure of $\mathrm{Ag}_{2} \mathrm{~S}: c_{\text {ion }} \gg c_{\text {eon }}, c_{\text {ion }} \sim$ constant, $c_{\text {eon }} \propto a_{\mathrm{Ag}}[5,7,13]$, and is thus consistent with the space-charge polarization characteristics represented by Eq. 4. However, the absolute capacitance value is far too high: $10^{4}$ times the value estimated using the electron concentrations of $\mathrm{Ag}_{2} \mathrm{~S}$ [5].

According to the diffuse double layer theory, the screening length becomes sub-nm, which cannot be described by the continuum model for the space-charge polarization. It should be noted that a similarly high interfacial capacitance of the blocking electrode was observed [21-23], suggesting that this phenomenon may be quite general.

The high capacitance value, exceeding $\mathrm{mF} / \mathrm{cm}^{2}$, can be ascribed to the adsorption capacitance at the core of the interface. It should be noted that the adsorption capacitance is connected not in series but in parallel with the doublelayer capacitance [28]. The adsorption capacitance should be distinguished from the parallel plate capacitance between the electrode surface and the inner Helmholtz layer or between the inner Helmholtz layer and the outer Helmholtz layer, which are connected in series with the diffuse double layer. The latter can be as large as a few tens of $\mu \mathrm{F} / \mathrm{cm}^{2}$ but, connected in series to the double layer capacitance, cannot account for the high interfacial capacitance value observed presently. 
Fig. 3 Fit parameters or material properties obtained by generalized equivalent circuit analysis
Partial Conductivities
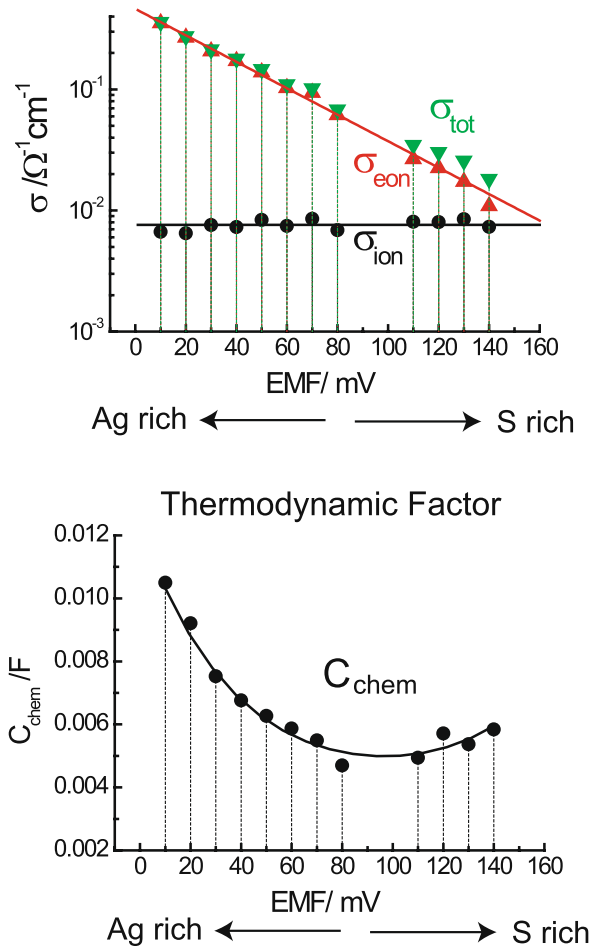

Interfacial Capacitance

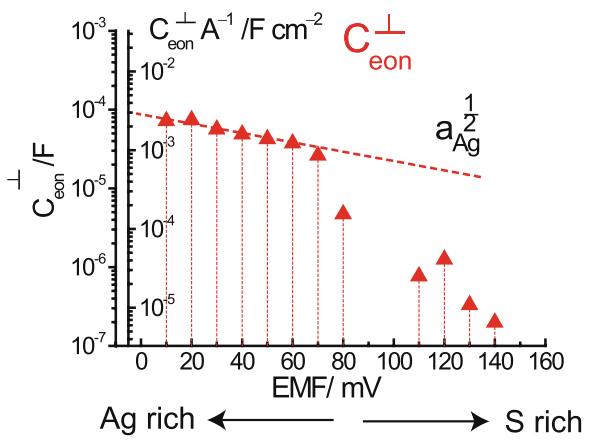

Transference Numbers
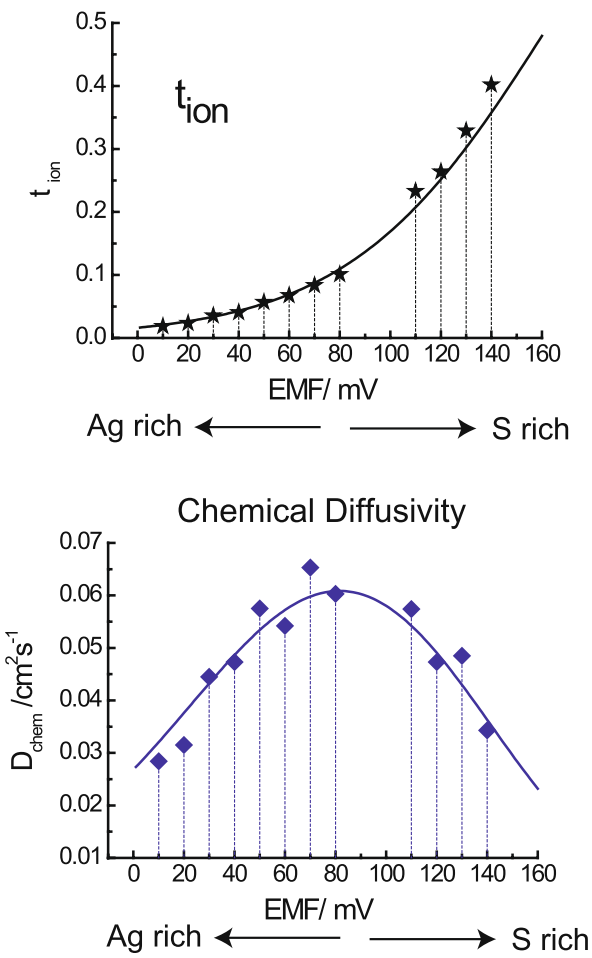

Blocking Electrodes

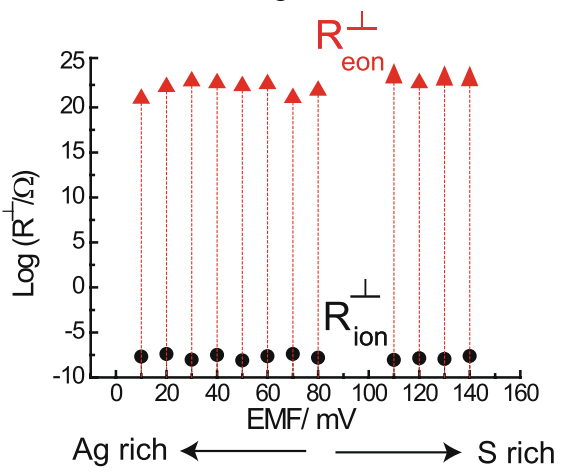

If one considers a simple Langmuir isotherm, the adsorption capacitance is proportional to the bulk activity of the adsorbed species. Thus $C_{\text {eon }}^{\perp}$ should be proportional to $c_{\text {eon }}$, which is in turn proportional to $a_{\mathrm{Ag}}$. The experimental observation of $C_{\mathrm{eon}}^{\perp} \propto \sqrt{\mathrm{a}_{\mathrm{Ag}}}$ is not consistent with this. The exact character of the interfacial capacitance should be further investigated.

When $R_{\text {ion }} \gg R_{\text {eon }}$, in other words, if the unblocked carrier resistance is much higher than the blocked carrier one, it has been shown that Eq. 1 simplifies to the Randles equivalent circuit (Fig. 4a) [19], characterized by the interfacial capacitance from the blocked charge carriers connected "in parallel" with the simple Warburg impedance $Z_{W}$,
$Z_{W} \approx\left(R_{\text {ion }}-R_{\infty}\right) \frac{\tanh \sqrt{\frac{i \omega L^{2}}{4 D_{\text {chem }}}}}{\sqrt{\frac{i \omega L^{2}}{4 D_{\text {chem }}}}}$.

With the knowledge that the parallel capacitance does not originate from a simple diffuse double layer but rather adsorption or chemical effects at the core, the Randles-type circuit can be rather conveniently employed using commonly available fitting programs, e.g., Zview (Scribner Assoc. Inc., USA). The conditions for simplification appear to be satisfied for the experimental data in the present work when $\mathrm{EMF}=10 \mathrm{mV}$, for which $R_{\text {ion }}=7,380 \Omega$ and $R_{\text {eon }}=139 \Omega$ (Fig. 3). The fitting results for the modeled elements are consistent with those obtained using the 
Fig. 4 a Simplified equivalent circuit of Fig. 1 when the unblocked ion resistance is much higher than that of the blocked electron resistance $\left(R_{\text {ion }} \gg R_{\text {eon }}\right)$. b Simplified circuit applies when the chemical capacitance is much larger than the interfacial capacitance of the blocked carriers $\left(C_{\text {chem }} \gg C_{\text {eon }}^{\perp}\right)$
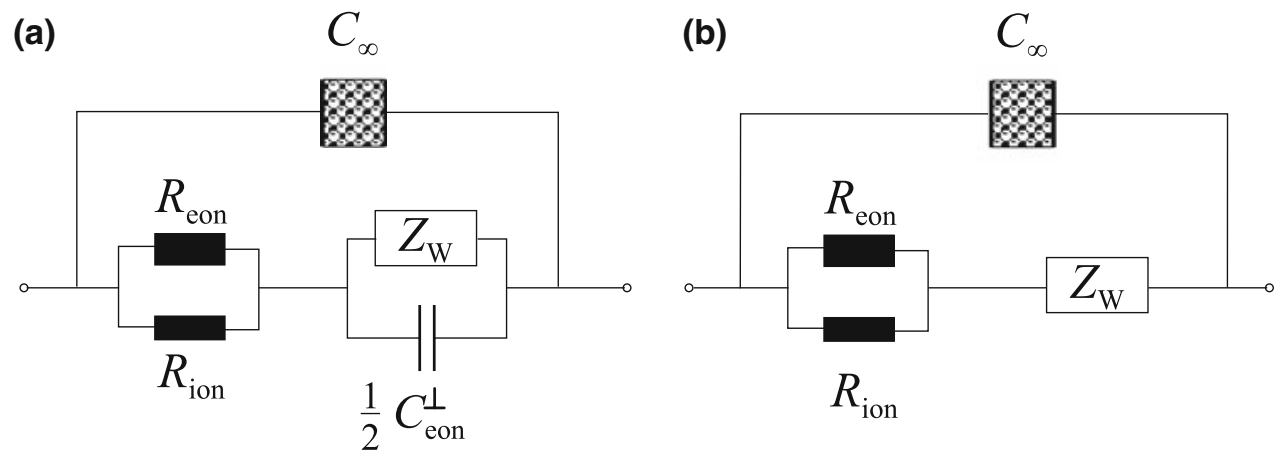

generalized equivalent circuit (Table 1), and the simulated spectrum is essentially the same as the one obtained with the generalized equivalent circuit shown in Fig. 2a (see also Fig. 6, discussed later).

Figure 5 represents the fit errors of the bulk resistance $R\left(=\left(R_{\text {ion }}^{-1}+R_{\text {eon }}^{-1}\right)^{-1}\right)$ and the parameters of the Warburg impedance $Z_{W}=R \tanh \sqrt{i \omega T} / \sqrt{i \omega T}$ in the circuits of Fig. $4 \mathrm{a}, \mathrm{b}$, and the parallel capacitance $C_{\mathrm{eon}}^{\perp}$ in the circuit Fig. 4b. The inappropriateness of the parallel capacitance $C_{\mathrm{eon}}^{\perp}$ with increasing EMF is clearly indicated: the errors exceed $10 \%$ for $\mathrm{EMF}=80 \mathrm{mV}$ and diverge $\left(>10^{7} \%\right)$ for $\mathrm{EMF}=130$ and $140 \mathrm{mV}$. For those data, the fit is actually better without $C_{\mathrm{eon}}^{\perp}$. As indicated in the right bottom corner of Fig. 5, much smaller errors are associated with the simple Warburg model in Fig. 4b than with Randles' model, although the fit values of the common elements are the same for both models. The situation is reversed in the case of $E M F=10 \mathrm{mV}$, as shown in the bottom left corner.

The equivalent circuit of Fig. $4 \mathrm{~b}$ was shown to be a good approximation of the generalized equivalent circuit if $C_{\text {chem }} \gg C_{\text {eon }}^{\perp}[19]$. This condition appears to be satisfied for high EMF values according to the fit results obtained for the generalized equivalent circuit (Fig. 3). The parameters included in the model of Fig. 4b are also consistent with the results obtained with the generalized circuit, and the simulated spectra are essentially the same (not shown). Note that the divergence in the estimation errors for $C_{\text {eon }}^{\perp}$ in Fig. 5 correlates with the sudden decrease in $C_{\text {eon }}^{\perp}$ values in Fig. 3 .

The simple Warburg impedance model in Fig. 4b has been used for most Hebb-Wagner polarization experiments [15, 18, 21, 25]. Unlike Randles' model with diverging errors for parallel capacitance when the condition is not justified, the simple Warburg model that does not consider the parallel capacitance works over the entire EMF range of the measurements, producing somewhat higher errors only in the low-EMF region; see Fig. 5. Figure 6 for $\mathrm{EMF}=10 \mathrm{mV}$ indicates significant discrepancies resulting from this neglect of the parallel capacitance, however. While the results obtained from the generalized equivalent

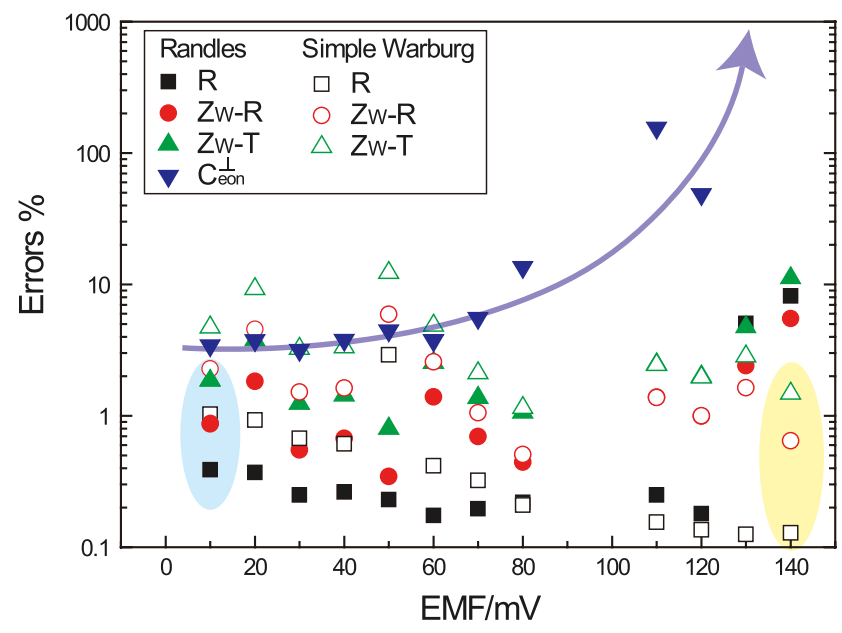

Fig. 5 Fit errors of the bulk resistance $R\left(=\left(R_{\text {ion }}^{-1}+R_{\text {eon }}^{-1}\right)^{-1}\right)$ and the parameters of the Warburg impedance $Z_{W}=R \tanh \sqrt{i \omega T} / \sqrt{i \omega T}$ in the circuits of Fig. $4 \mathrm{a}, \mathrm{b}$, and the parallel capacitance $C$ in the circuit Fig. 4b

circuit and the Randles-type approximation are indistinguishable, the simple Warburg model (Fig. 4b) shows some marked differences. As well as the small discrepancy in the low frequency behavior and in the dc limit (also shown in the inset), the substantial difference observed at higher frequencies $(f>0.1 \mathrm{~Hz})$ in the Bode plot should be noted. The inadequacy of simple Warburg impedance in this frequency range is equivalent to the inapplicability of $\sqrt{t}$-dependent short-time diffusion solutions in the dc relaxation experiment for the Hebb-Wagner cell, since they are related to each other by a Fourier-Laplace transformation $[15,17,18]$. The presence of a capacitive element in parallel with the Warburg-like constant phase element was also clearly demonstrated in a Hebb-Wagner cell configuration consisting of Li-conducting perovskites with platinum electrodes that selectively blocked ions [24].

The success of the simplified circuits in the extreme cases suggests that the other elements in the generalized equivalent circuit do not contribute to the overall impedance appreciably in those particular cases, and are thus possibly associated with large errors in fitting, although no 


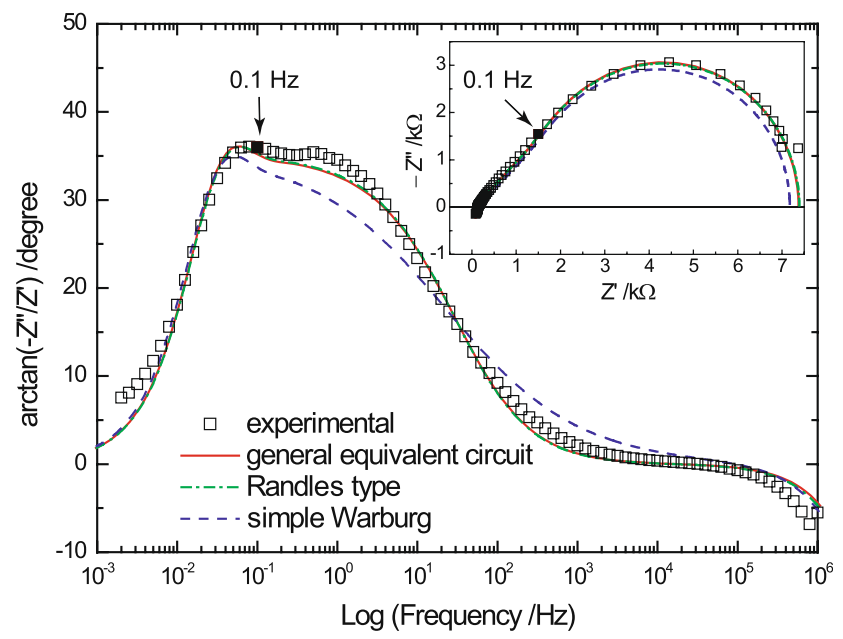

Fig. 6 Bode plot of phase angle of impedance with EMF $=10 \mathrm{mV}$, showing the fit results obtained using the generalized equivalent circuit (Fig. 1) and simplified circuits of Randles' type (Fig. 4a) and of simple Warburg type (Fig. 4b). Impedance plots are shown in the inset

individual error estimation is given at the moment. However, this situation is commonly encountered in the electrochemical characterization of various materials systems. When the respective impedance elements change characteristically with experimental variables, there are situations in which the responses severely overlap and some elements are manifested to a lesser degree. As long as the model is physically based, the fit parameters (even when they have large errors) can be related to material parameters. Thus, a robust impedance model should be established over the range of the experimental variables. As the current work beautifully demonstrates, the generalized complete equivalent circuit can be employed to systematically assess a wide range of material parameters. This not only improves material parameter accuracy but also yields information that is exhaustive.

It should be emphasized that in the present analysis we only used ideal capacitors and thus ideal Warburg elements in the equivalent circuits of the generalized and simplified cases as shown in Figs. 1 and 4. Employing constant phase elements $Z=Q^{-1}(i \omega)^{-n}$ with $n \lesssim 1$ (and $n \lesssim 0.5$ for the Warburg case) usually improves the overall agreement between the experimental and fitted spectra [21-23].

\section{Conclusion}

The generalized equivalent circuit of a mixed conductor comprising space-charge polarization as well as stoichiometry polarization has been successfully applied to the model mixed conductor $\mathrm{Ag}_{2} \mathrm{~S}$ with selectively blocking electrodes in the Hebb-Wagner configuration of $\mathrm{Ag}|\mathrm{AgI}| \mathrm{Ag}_{2} \mathrm{~S}|\mathrm{AgI}| \mathrm{Ag}$. Due to the ideal behavior of $\mathrm{AgI}$ as a blocking electrode under the experimental conditions applied, and the widely variable thermodynamic and kinetic properties of the mixed conducting $\mathrm{Ag}_{2} \mathrm{~S}$, the validity of the generalized and simplified equivalent circuits can be clearly demonstrated. The interfacial capacitance was found to be too large to be explained by the diffuse double layer capacitance, and was ascribed to the adsorption capacitance at the core of the interface, which should be connected in parallel with the double-layer space-charge polarization.

Acknowledgments This work was financially supported by the Korea Research Foundation Grant funded by the Korean Government (MOEHRD) (KRF-2008-531-D00006).

\section{References}

1. Terabe K, Hasegawa T, Nakayama T, Aono M (2005) Nature 433:47-50

2. Faraday M (1833) Philos Trans R Soc 123:507-515

3. Tubandt C, Schibbe G (1921) Z Anorg Chern 117:1-47

4. Wagner C (1933) Z Phys Chem 21:42-47

5. Miyatani S (1955) J Phys Soc Jpn 10:786-793

6. Bonnecaze G, Lichanot A, Gromb S (1978) J Phys Chem Solids 39:299-310

7. Becker KD, Schmalzried H, von Wurmb V (1983) Solid State Ion 11(3):213-219

8. Ding S, Petuskey WT (1992) Solid State Ion 58(1-2):123-132

9. Hebb MH (1952) J Chem Phys 20:185-190

10. Wagner C (1953) J Chem Phys 21(10):1819-1827

11. Wagner C (1975) Prog Solid State Chem 10:3-16

12. Yokota I (1961) J Phys Soc Jpn 16:2213-2223

13. Schmalzried H (1980) Prog Solid State Chem 13:119-157

14. Maier J (1984) Z Phys Chem N F 140:191-215

15. Lee JS, Yoo HI (1994) Solid State Ion 68:139-149

16. Jamnik J, Maier J (1999) J Electrochem Soc 146(11):4183-4188

17. Lee JS (1994) Measurement of partial ionic conductivity of electronic conductor oxides via AC impedance spectroscopy combined with DC relaxation. Ph.D. thesis, Seoul National University

18. Lee JS, Yoo HI (1995) J Electrochem Soc 142(4):1169-1176

19. Jamnik J, Maier J, Pejovnik S (1999) Electrochim Acta 44:4139_ 4145

20. Jamnik J, Maier J (2001) Phys Chem Chem Phys 3:1668-1678

21. Lai W, Haile SM (2005) J Am Ceram Soc 88:2979-2997

22. Fleig J, Kim HR, Jamnik J, Maier J (2008) Fuel Cells 08(5):330 337

23. Baumann FS, Fleig J, Habermeier HU, Maier J (2006) Solid State Ion 177(11-12):1071-1081

24. Lee JS, Yoo KS, Kim TS, Jung HJ (1997) Solid State Ion 98:1526

25. Andreaus R, Sitte W (1997) J Electrochem Soc 144(3):10401044

26. Barsoukov E, Macdonald JR (eds) (2005) Impedance spectroscopy: theory, experiment, and applications, 2nd edn. Wiley, London, p 197

27. Song CR, Yoo HI (2000) Phys Rev B 61:3975-3982

28. Macdonald JR, Franceschetti DR, Lehnen AP (1980) J Chem Phys 73:5272-5293 